"A second-step splicing activity is conserved from yeast to human" Cicarelli, R.M.B., Khaouja, A., Codony, C., Espuny, R., Eritja, R., Bach-Elias, M. Biochem. Biophys. Res. Comm., 247(2), 204-206, (1998). doi: 10.1006/bbrc.1998.8771

\title{
A Second-Step Splicing Activity Is Conserved from Yeast to Human
}

\author{
R. M. B. Cicarelli, ${ }^{1}$ A. Khaouja, C. Codony, R. Espuny, R. Eritja, ${ }^{2}$ and \\ M. Bach-Elias ${ }^{3}$ \\ CID-CSIC, c/Jorge Girona Salgado 18-26, 08034 Barcelona, Spain \\ ${ }^{1}$ Present address: UNESP, Faculdade de Ciencias Farmaceuticas, \\ Araraquara, SP, Brazil \\ ${ }^{2}$ Present address: EMBL, Meyerhofstrasse 1, Postfach 10.2209, Heidelberg, \\ Germany \\ ${ }^{3}$ To whom correspondence should be sent. Email: mbebmc@cid.csic.es
}

\begin{abstract}
We describe a defective HeLa nuclear extract which is particularly deficient in step 2 of splicing reaction. With this extract we have studied the conservation of a second-step activity from yeast to human cells. We detected a $S$. cerevisiae second-step splicing activity that allows restoration of step 2 of the defective HeLa nuclear extract, which indicates that yeast purified fraction has a second-step activity that is conserved from yeast to human cells. The activity is a yeast UsnRNP protein(s) since it is purified with anti-tri-methyl-guanosine by immunoaffinity columns.

The splicing reaction of nuclear pre-mRNAs has a similar mechanism in yeast and mammalian cells (1). Human spliceosomal factors can be divided in three categories: 1) UsnRNPs; 2) SR proteins and 3) other polypeptides (2). In yeast no SR proteins have been detected (3). UsnRNPs are the most conserved factors from yeast to mammalian cells, consisting of complexes of U1, U2, U4/U6 and U5 snRNAs with a known set of proteins (4). In this study we attempt to determine which second-step activities are conserved from yeast to human cells. We used an in vitro complementation in which yeast spliceosomal proteins were added to a HeLa cell nuclear extract defective for the second step reaction. The study focuses on a second-step activity present in a yeast UsnRNP splicing particle, which is conserved across species. Yeast UsnRNPs were chosen because a) they are easy to purify with immunoaffinity columns and b) many yeast second-step factors have been cloned and can be easily purified from recombinant cells. It is thus possible to perform further studies to improve our knowledge of second-step splicing activities.

UsnRNP splicing factors from yeast source contain a second-step activity that complements in vitro the second-step splicing reaction of the defective mammalian system.
\end{abstract}

MATERIALS AND METHODS 
HeLa nuclear extracts defective for the second step ( $\left.h N E^{-2 n d}\right)$. We obtained nuclear HeLa extracts from frozen cells defective for the second step of the splicing reaction. Frozen HeLa cells, provided by $4 \mathrm{C}$ Company (Computer Cell Culture Center S.A., Mons, Belgium) were used for the preparation of extracts. The cells were stored at $-80{ }^{\circ} \mathrm{C}$ for $6-8$ months. The method of Dignam et al. (5) was modified on the following steps: a) the cells were defrosted in one volume of on the following steps: a) the cells were defrosted in one volume of PBS buffer $(20 \mathrm{mM}$ potassium phosphate $\mathrm{pH} 7.3,130 \mathrm{mM} \mathrm{NaCl}$ ) at $37{ }^{\circ} \mathrm{C}$ (this temperature is only used to thaw the cells; all other steps were performed at $\left.4{ }^{\circ} \mathrm{C}\right)$; b) during the swell step in buffer A (see composition of this buffer in ref. 5), cells were allowed to swell on ice for $30 \mathrm{~min}$. The swollen cells were collected by centrifugation as stated and resuspended in two cell pellet volumes (i.e., the volume before the initial wash with buffer A) of buffer A and lysed by 15 strokes of a glass Dounce homogenizer (tight pestle); c) the extraction of the nuclei in buffer C (see composition of this buffer in ref. 5), which was done by 15 strokes (instead of 10 strokes) of a glass Dounce homogenizer. In summary, the main changes on the procedure are: use of frozen cells stored at $-80{ }^{\circ} \mathrm{C}$ for 6-8 months; longer incubation in hypotonic buffer and increase of the stroke number in all Douncer homogenizer steps. Frozen HeLa cells swelled little in hypotonic buffer $A$. This defect provokes that few protein is extracted from the total pellets obtained, which is mainly composed by not swollen and unbroken cells (this observation was followed under microscope and protein determination). We therefore decided to modify this swelling step to obtain a higher yield of nuclei.

One of these extracts is shown in Fig. 1. Lane 1 corresponds to the splicing reaction in a fully active nuclear extract (from HeLa cells harvested from cell culture media and immediately used -referred as fresh cells-to obtain nuclear extracts), in which the lariat-exon2 and exon1 intermediate, and the exon1-exon2 product are clearly shown. Lanes 2-5 in Fig. 1 show the splicing reaction for HeLa cell nuclear extract defective for the second step ( $\mathrm{hNE}^{-2 \mathrm{nd}}$ ); the control sample has no ATP (lane 2) and the other samples have increasing percentages of $\mathrm{hNE}^{-2 n d}$ (between $20-60 \%$ of nuclear extract in lanes 3-5). Lanes 3-5 show that the first step of the splicing reaction is accomplished in these extracts (lariat still bound to exon2), but not the second step (absence of the exon1-exon2 product in lanes 3-5). The RNA bands shown in the figure 1 were characterized and correspond to the wild type intermediates. Three different $\mathrm{hNE}^{-2 \mathrm{nd}}$ were obtained with this method, and the experiments here shown were repeated with these three extracts with similar results.

Purification of yeast UsnRNPs (yUsnRNPs). UsnRNPs were purified from total yeast extracts (prepared as previously described, (6)) passed through anti- $m_{3} G$ immunoaffinity columns containing either the anti- $m_{3} G$ monoclonal antibody called Ab-1 (Oncogene Science, ref 7). The immunoaffinity purification of the yeast UsnRNPs from total yeast extract was described by Fabrizio, P. et al. (6). yUsnRNPs were concentrated either by PEG 40.000 or by centrifugation through $3 \mathrm{kDa}$ salt-cut membranes and finally dialyzed against PBS. The yeast strain used to obtain the total yeast extract was EJ101 (generous gift from Dr. M. Rosbash). 
Splicing reactions. Splicing conditions were those previously published (8).

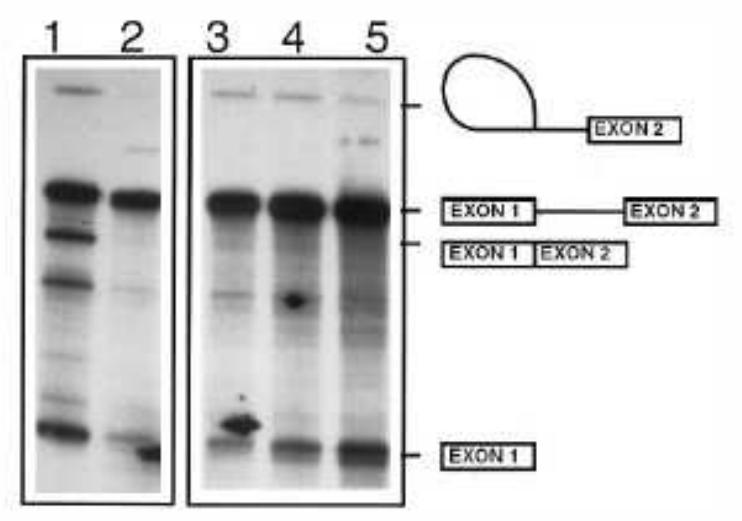

FIG. 1. Splicing reaction of defective and fully active extracts with $\beta$-globin premRNA. The defective HeLa nuclear extract $\left(\mathrm{hNE}^{-2 n d}\right)$, which only performed the first step of the splicing reaction, was prepared as described in Material and Methods. The fully active extract was prepared as previously described (5). Lanes: 1 , fully active extract $(40 \%)$ with ATP; lane $2, \mathrm{hNE}^{-2 n d}(40 \%)$ without ATP; lanes 3-5, hNE 2nd with ATP using $20 \%, 40 \%$ and $60 \%$ of $\mathrm{hNE}^{-2 \mathrm{nd}}$, respectively. The total time of the splicing reaction was 90 min.

\section{RESULTS AND DISCUSSION}

Extracts depleted for second-step factors are suitable to perform complementation studies with functional factors, particularly with those from other sources. This study focuses on one group of spliceosomal factors: the yeast UsnRNPs, which were purified in a native form by binding to anti- $m_{3} G$ immunoaffinity columns and elution with competitor nucleoside (6). The rationale for using yeast UsnRNPs was discussed in the introduction. $\mathrm{hNE}^{-2 n d}$ extracts were complemented with purified yUsnRNPs. In these complementation experiments, yUsnRNPs were added to $\mathrm{hNE}^{-2 \mathrm{nd}}$ at different times for $90 \mathrm{~min}$ following initiation of the splicing reaction. The results are shown in Fig. $2 A$ with the $\beta$-globin pre-mRNA. Lanes 1-3 corresponds to splicing reactions with a fully active extract; two duplicate reactions with ATP (lanes 2 and 3 ) and one control reaction without ATP (lane 1 ). Lanes $4-10$ are splicing reactions with $\mathrm{hNE}^{-2 \mathrm{nd}}$ complemented (lanes 6-10) with $1 \mu \mathrm{g}$ of yUsnRNPs at 0, 15, 30, 45 and $60 \mathrm{~min}$ after the initiation of the splicing reaction respectively, while lanes 4 and 5 are negative controls without yUsnRNPs (and without and with ATP, respectively). Lane 5 shows that only the first step is completed in $\mathrm{hNE}^{-2 \mathrm{nd}}$ splicing reaction, as demonstrated by the presence of the branched intronexon2 intermediate and by the absence of the debranched intron and exon1-exon2 products, this exon1-exon2 band was characterized and it corresponded to the same wild-type product shown in Fig. 2A, lane 2 and 3. $40 \%$ of extract and rabbit $\beta$-globin pre-mRNA was used in all these splicing reactions. Out of this figure we can extract the following observations. a) Lanes 7 and 8 of Fig. $2 \mathrm{~A}$ indicate that when $1 \mu \mathrm{g}$ of purified yUsnRNPs was added to $\mathrm{hNE}^{-2 \mathrm{nd}} 15$ or $30 \mathrm{~min}$ after the initiation of the reaction, the second 
step was completed (note exon1-exon2 and debranched intron in lanes 7 and 8 ). The second step was not completed when buffer was added instead of yUsnRNPs (Fig. 2A lane 5) or when yUsnRNPs were added 0,45 or 60 min after the initiation of the splicing reaction (the second step is indicated by the presence of exon1-exon2, which does not appear in lanes 4-6, 9 or 10).

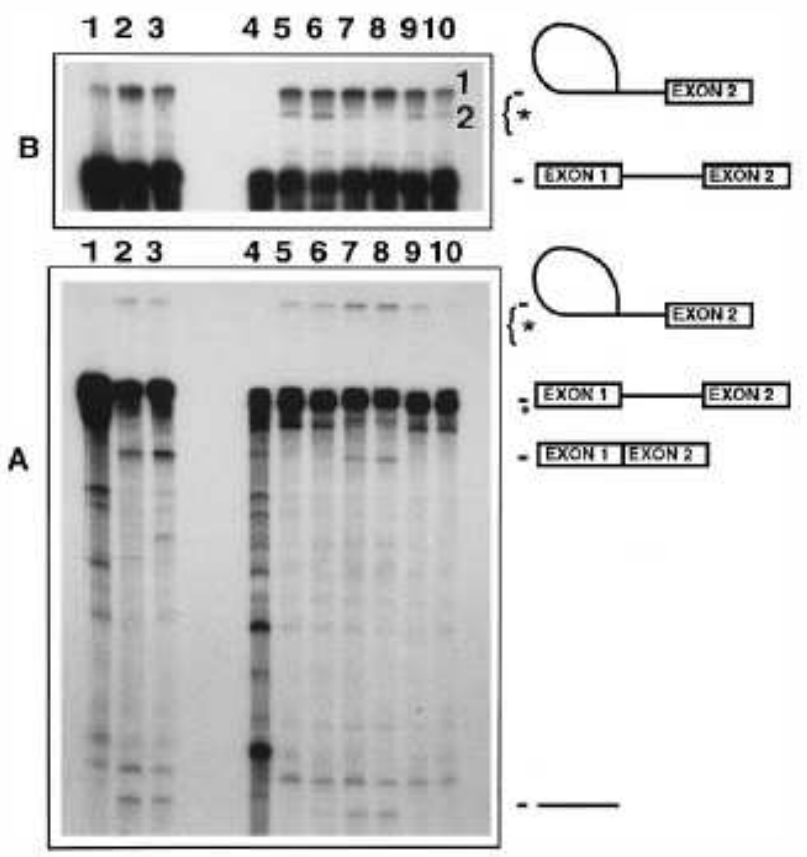

FIG. 2. Complementation of the $\mathrm{hNE}^{-2 n d}$ with yUsnRNPs by time-course performance. Panel $B$ shows a longer time exposition of the upper part of the gel shown in Panel A. All the reactions, which were done with $\beta$-globin pre-mRNA, were incubated for a total time of $90 \mathrm{~min}$ and with $40 \%$ of extract. Lanes 1-3 are splicing reaction control samples with fully active extract and ATP (lanes 2 and 3, duplicate reactions) and without ATP (lane 1 ). Lanes 4 and 5 are splicing reaction control samples of $\mathrm{hNE}^{-2 \text { nd }}$ without and with ATP, respectively. In lanes 6-10, the splicing reactions were complemented with $1 \mu \mathrm{g}$ of yUsnRNPs at $0,15,30,45$ and $60 \mathrm{~min}$, respectively, after the beginning of the reaction and allowed to proceed until a total incubation time of $90 \mathrm{~min}$. The region indicated with a bracket and an asterisk corresponds to the additional intermediates obtained in splicing reactions performed with $\mathrm{hNE}^{-2 n d}$; they are better shown in the upper part of panel $\mathrm{B}$. Band 1 in panel $\mathrm{B}$ turned out to be the wild-type lariat which contains the wild-type $5^{*}$ splice-site and branch-point. Band 2 in panel B could not clearly be characterized.

This complementation depends on yUsnRNPs, since other protein fractions (e.g. DEAE I and II from Lindsey et al. see ref (9)) or buffers (buffer A or D from Dignam et al., see ref. (5)) did not complement hNE-2nd to complete the second step products. An increase of the amount of hNE-2nd (60-90\% of extract during the splicing reaction; $60 \%$ extract is shown in Fig. 1, lane 5) did not allow the completion of the second step. The addition of $1 \mu \mathrm{g}$ of total protein of fully active nuclear extract from yeast was not enough to complete the second step reaction (not shown). This indicates that the complementation to the second-step product is dependent on the 
presence of purified yUsnRNPs and it is not dependent on the presence of any protein.

We conclude that there is a conservation of a second-step activity from yeast to human cells that belongs to the UsnRNP family. This activity is named ySSA (yeast second-step activity), to distinguish it from the previously described SSF1 yeast factor (11).

Splicing reactions in $\mathrm{hNE}^{-2 \mathrm{nd}}$ showed additional bands near the branched intron which are marked with asterisks in Figs. $2 \mathrm{~A}$ and $2 \mathrm{~B}$ (the upper half of Fig. 2A, containing the lariats, was exposed longer time; see Fig. 2B). Since these additional bands could be aberrant lariats or degradation products, they were studied with reverse-transcriptase and oligonucleotide primers which were complementary to sequences near and downstream of the branch-point or $5^{*}$ splice-site. Band marked as " 1 " in Fig. 2B turned out to be the wild-type lariat that uses the wild-type $5^{*}$ splice-site and branch-point of the $\beta$-globin pre-mRNA. Unfortunatelly, we are unable to characterize the band marked as " 2 " in Fig. $2 B$, since only low amounts from band 2 could be purified and there is a high background in this area. The product exon1-exon2 seen in Fig. 2 lanes 7 and 8 was characterized as the wild-type product as follows: a) in previous works, this band was already characterized as the wild-type product in fully active extracts (see ref. 8); b) the band named exon1-exon 2 in Fig. 2 lanes 7 and 8 have the same mobility as the exon1-exon 2 band obtained in fully active extracts (compare lanes 7 and 8 with lanes 2 and 3 ); c) the band has a molecular weight of 301 nucleotides that would agree with the exon1-exon 2 molecular weight and d) the band was assayed by RT-PCR and showed to be the exon1-exon 2 product (not shown). The band marked as the debranched intron in lanes 7 and 8 was previously analyzed (8) and it was assumed to be this product as it was obtained linked to exon1-exon2 product (see lanes 7 and 8) and had a molecular mass of 126 nucleotides.

There was a small time-course window, between 15 and $30 \mathrm{~min}$ after the initiation of the reaction, when yUsnRNPs complemented and completed the second step (Fig. 2B, see exon1-exon2 band in lanes 7 and 8). Outside this window, no second-step complementation was detected despite the presence of yUsnRNPs (Fig. 2B, lanes 6, 9 and 10). This observation indicates that the complementation is better achieved during the time lag, after 30 min of in vitro reaction. Finally, we want to remark that the ySSA activity could be a combination of different yeast UsnRNPs or yeast UsnRNPs proteins.

Due to the fact that very few amounts of yUsnRNPs can be obtained from yeast extracts, the assay here described will be more suitable to complementation with recombinant yeast proteins than with fractions obtained directly from yUsnRNPs. Yeast second-step factors Prp18p, Slu7p and Prp17p seem to associate with U5snRNP (3). There is also an uridine recognition defect caused by the prp8-101 mutation that inhibites the second step splicing reaction (12). Prp8p is the protein that presents a more stable association with U5snRNA (3). So far, Prp16p has not been detected bound to any UsnRNP, but as Prp16p interacts with Prp17p and Slu7p, it can be close or even be loosely bound to the U5snRNP (10). So far, SSF1 has been not described to be associated with any yeast UsnRNP particle (11). Our very preliminar results indicate that partially purified Slu7p and Prp16p, or a combination of Slu7p+Prp16p, do not complement the defective extract 
here described. Any of these factors, or a combination of some of them, could be the splicing activity here detected, and the complementation with the each recombinant protein would better answer this question.

\section{ACKNOWLEDGMENTS}

We thank J. M. Barrios, X. Jimenez and R. Perez for technical assistance, and all members of the $M$. Bach-Elias group for the comments about the manuscript. This work was supported by PGC Grant No. PB920004 and an Alexander von Humboldt Foundation grant. C. Codony was the recipient of a fellowship from PGC; R.B. Cicarelli was the recipient from a fellowship from CAPES and Spanish MEC; A. Khaouja was the recipient of a fellowship from the Moroccan government.

\section{REFERENCES}

1. Sharp, P. A. (1994) Cell 77, 805-815.

2. Kramer, A. (1996) Ann. Rev. Biochem. 65, 367-409.

3. Beggs, J. D. (1995) in Pre-mRNA Processing (Lamond, A. I., Ed.), pp. 79-95, R.G. Landes Company.

4. Bach, M., Bringmann, P., and Lührmann, R. (1990) RNA Processing. Part

B. Specific Methods 181, 232-257.

5. Dignam, J. D., Lebovitz, R. M., and Roeder, R. G. (1983) Nucl. Acids Res. 11, 1475-1489.

6. Fabrizio, P., Esser, S., Kastner, B., and Lührmann, R. (1994) Science 264, 261-265.

7. Krainer, A. R. (1988) Nucleic Acids Res. 16, 9415-9429.

8. Winkelmann, G., Bach, M., and Lührmann, R. (1989) EMBO J. 8, 31053112.

9. Lindsey, L. A., Crow, A. J., and Garciablanco, M. A. (1995) Journal of Biological Chemistry 270, 13415-13421.

10. Jones, M. H., Frank, D. N., and Guthrie, C. (1995) Proc. Natl. Acad. Sci. USA 92, 9687-9691.

11. Ansari, A., and Schwer, B. (1995) EMBO J. 14, 4001-4009.

12. Umen, J. G., and Guthrie, C. (1995) Genes Develop. 9, 855-868. 